\title{
Técnicas auxiliares para el diagnóstico de caries incipiente interproximal en molares deciduos de niños de 4 a 10 años
}

\author{
Auxiliary techniques for the diagnosis of incipient interproximal caries, in deciduous molars \\ of children from 4 to 10 years old
}

\author{
Jessica Pardo Salas ${ }^{1 a}$, Tannya Valarezo Bravo ${ }^{1 a}$
}

\section{RESUMEN}

Objetivo: La lesión incipiente de caries conocida por sus características clínicas de aspecto blanco opaco tras el secado, con superficie rugosa y áspera, es la primera evidencia visible de actividad cariogénica, observada en fosas y fisuras, superficies proximales cerca del punto de contacto y a lo largo del margen gingival, en relación con las superficies proximales por la difícil accesibilidad, la inspección visual se ve obstaculizada, por lo que el objetivo de este estudio es identificar la técnica auxiliar de caries incipiente interproximal con mejores resultados para el diagnóstico en molares deciduos. Materiales y método: Se realizó un estudio de tipo descriptivo y transversal. La muestra fue compuesta por 50 niños de 4 a 10 años de edad. Se registró en el odontograma información del diagnóstico aplicado, tomando en cuenta los criterios según ICDAS 2005. El examen se realizó en diferentes sesiones, realizando previamente una profilaxis, para el estudio se utilizó espejo bucal, lupa de aumento tipo viscera $(3,5 X)$, lámpara LED y radiografías aleta de mordida.. Resultados: Se encontró una diferencia significativa en la eficacia para la detección de lesiones cariosas incipientes interproximales entre las técnicas evaluadas. Conclusión: La técnica de magnificación 3,5X permitió detectar mayor número de lesiones cariosas incipientes interproximales en molares deciduos con un total de 69. Seguida por la visual simple y la radiográfica, siendo la transiluminación la que presentó el menor nivel de eficacia.

Palabras Clave: Caries dental; Magnificación; Radiografia; Transiluminacion. (Fuente: DeCS BIREME)

\section{ABSTRACT}

Objective: The incipient carious lesion known for their clinical characteristics like white opaque after drying shows a rough surface, this is the first evidence of caries which is actively and visible on the surface of the dental tissue observed in a pit and fissure, proximal surfaces near the contact point along the gingival margin, in relation to the proximal surfaces by having a difficult accessibility, just by visual inspection is a big obstacle, so the objective of this study is identify the auxiliary a proximal decay incipient technique with improved an outcome to diagnosis in primary molars. Materials and Methods: A transversal and descriptive study was performed. The sample was composed by 50 children from 4-10 years old. A medical history was applied, which recorded all the dental information about the diagnosis, taking into account the criteria according to ICDAS in 2005. The examination was took place various sessions, a prophylaxis was previously performed, for this study we used an oral mirror, magnifying glass, a type of viscera which increased (3.5X), LED lamp and per apical bitewing x-rays. Results: A significant difference in efficacy for detection of interproximal caries lesions among emerging techniques evaluated were found. Conclusion: A 3.5X magnification technique made it possible to detect more caries lesions in primary molars with a total of 69. Followed by visual and radiographic being Trans illuminations which had the lowest levels of effectiveness.

Keywords: Dental caries; Magnificación; Radiography; Transillumination. (Source: MeSH NLM)

Recibido: 06 de setiembre de 2018

Aprobado: 31 de octubre de 2018

Publicado: 30 de diciembre de 2018

${ }^{1}$ Universidad de Loja, Ecuador ${ }^{2}$ Universidad

adontóloga.

Correspondencia:

Jessica Pardo Salas

Correo electrónico: jessimar_1403@hotmail.com
Este es un artículo Open Access distribuido bajo la licencia Creative Commons Atribución-NoComercial- Compartir Igual 4.0

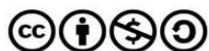

Citar como: Jessica Pardo Salas, Tannya Valarezo Bravo. Técnicas auxiliares para el diagnóstico de caries incipiente interproximal en molares deciduos de niños de 4 a 10 años. KIRU. 2018; 15(4): 159 - 165. https://doi.org/10.24265/kiru.2018.v15n4.01 


\section{INTRODUCCIÓN}

La lesión inicial, denominada mancha blanca, es la primera evidencia visible de actividad cariogénica en la superficie del tejido dentario. Esta evidencia es la consecuencia del acúmulo de numerosos episodios de desmineralización y mineralización. Esta lesión se caracteriza por presentar una superficie de esmalte intacto con un aspecto blanco opaco tras el secado, mostrando al tacto una superficie rugosa y áspera. (1)

La lesión de caries proximal se encuentra preferiblemente por debajo/encima del punto de contacto interdental y que se extienden en dirección cervical hasta el margen gingival debido a la mayor acumulación de biopelícula en esa zona.(2)

La exploración clínica comprende la inspección visual y la palpación, en la actualidad, se da mucha importancia a la inspección visual de las superficies dentarias bajo una buena fuente de luz, con limpieza y secado previo de estas superficies. Es aconsejable el uso de espejos de aumento o lentes binoculares de magnificación.(3)

\section{MATERIALES Y METODOS}

La presente investigación fue de carácter cuantitativa, descriptiva, transversal y prospectiva. El área de estudio en la que se realizó la investigación fue la Escuela de Educación Básica "Mons. Juan María Riofrío", el universo corresponde a 274 niños y niñas; y de forma aleatoria se tomó una muestra correspondiente a 50 niños/as de 4 a 10 años de edad.

Los criterios de inclusión fueron: niños/as que asistieron el día en el que se realizó el diagnóstico y que participaron en el mismo, niños/as comprendidos en edades de 4 a 10 años, con dentición decidua o mixta y con autorización pertinente de los padres de familia. Dentro de los criterios de exclusión: niños/as que no asistieron el día en que se realizó el diagnóstico, no colaboradores, mayores a 10 años y menores de 3 años y niños/as sistemáticamente comprometidos y de difícil acceso odontológico.

Los métodos utilizados fueron: la historia clínica, elaborada con información general y el respectivo odontograma basado en el sistema propuesto por la Federación dental Internacional (ICDAS 2005); y el respectivo consentimiento informado. Los instrumentos utilizados: consentimiento informado, historia clínica, set de diagnóstico espejo bucal, lámpara de luz halógena, lentes de aumento tipo viscera $3,5 \mathrm{X}$, lentes de protección de luz halógena y radiografías aleta de mordida.
Para la determinación de la técnica con mayor eficacia para el diagnóstico de caries incipiente interproximal en molares deciduos de los niños, se realizó en diferentes sesiones: se procedió a llenar la historia clínica; previamente se realizó una profilaxis, para eliminar la placa bacteriana que podría interferir en la aplicación posterior de las técnicas en los niños-as. Realizada la profilaxis se procedió al diagnóstico, examinando a cada niño en posición horizontal ubicado en el sillón dental, aplicando primeramente la técnica visual simple: la misma que fue realizada con iluminación artificial de la unidad dental, secando las superficies proximales de los molares deciduos por cinco segundos con jeringa de aire y el uso respectivo de espejo bucal plano $\mathrm{N}^{\circ} 5$ sin aumento y mediante los criterios establecidos por el ICDAS 2005.

Seguidamente se examinó mediante la técnica de magnificación, en las mismas condiciones en la que se llevó a cabo el examen visual, con ayuda de lentes de aumento tipo viscera 3,5X. Después se aplicó la técnica de transiluminación, realizando aislamiento relativo de los dientes con rollos de algodón en la boca del niño y con ayuda de una lámpara de luz halógena (LED) se colocó la punta de la fibra sobre las superficies interproximales de los dientes durante 10 segundos; durante este procedimiento se utilizó protectores oculares. $Y$ finalmente en la última sesión se aplicó la técnica radiográfica con aleta de mordida, la misma que se realizó en el laboratorio de radiología: las películas fueron identificadas por el nombre de cada niño y con la debida protección o nomas de bioseguridad, se colocó al niño confortablemente en la silla, con la parte posterior en posición vertical, y la cabeza colocada normalmente de modo que el plano de Camper sea paralelo al piso, y el plano medio sagital sea perpendicular al piso; seguidamente la película se colocó en la boca del niño paralela a las coronas de los dientes superiores e inferiores, colocando primeramente en el piso de la boca, centrando los dientes a radiografiar, posteriormente y sin soltar la aleta de mordida, se pidió al niño que muerda suavemente, cierre lenta y completamente la boca; y con la angulación vertical de $10^{\circ}$ positivo del cono del aparato de Rx gnatus, se disparó con un tiempo de exposición de 0,8 segundos. Luego se retiró la película radiográfica de la boca y posteriomente se reveló. Para el revelado de las películas $x$ se utilizó revelador y fijador AGFA DENTUS $125 \mathrm{ml}$, los mismos que se mezclaron con agua una vez reveladas, lavadas y secadas las mismas se colocaron en un porta radiografías para la posterior interpretación con ayuda de un negatoscopio. Los datos obtenidos mediante las cuatro técnicas aplicadas se registraron en la historia clínica.

Una vez obtenida la información registrada en la historia clínica, se tabuló mediante la utilización de un programa Excel, representando mediante tablas y gráficas y posterior discusión de los resultados obtenidos.

Los índices utilizados fueron el ICDAS (Sistema Intemacional Visual de Valoración y Detección de Caries Dental): 
Tabla 1. Códigos ICDAS en dentición primaria:

\begin{tabular}{|c|l|}
\hline 0 & SANO al secado con aire durante 5 segundos \\
\hline 1 & Mancha blanca marrón en esmalte seco \\
\hline 2 & Mancha blanca marrón en esmalte húmedo \\
\hline 3 & $\begin{array}{l}\text { Pérdida superficial de esmalte }<0,5 \mathrm{~mm} \text {. En esmalte } \\
\text { seco, sin dentina visible }\end{array}$ \\
\hline 4 & $\begin{array}{l}\text { Sombra oscura de dentina visible a través del } \\
\text { esmalte húmedo; con o sin pérdida superficial del } \\
\text { esmalte }\end{array}$ \\
\hline 5 & $\begin{array}{l}\text { Cavidad con dentina visible }>0,5 \mathrm{~mm} \text {. Hasta el } 50 \% \\
\text { de la superficie }\end{array}$ \\
\hline 6 & $\begin{array}{l}\text { Cavidad extensa más de } 50 \% \text { de la superficie } \\
\text { dentaria }\end{array}$ \\
\hline
\end{tabular}

\begin{tabular}{|c|l|}
\hline ICDAS & $\begin{array}{l}\text { Mancha blanca= incipiente } \\
\text { Rugosa-opaca= activas } \\
\text { Lisa-brillante= inactiva }\end{array}$ \\
\hline
\end{tabular}

Tabla 2. Códigos transiluminación ICDAS:

\begin{tabular}{|l|}
\hline No hay sombra o área manchada \\
\hline $\begin{array}{l}\text { La lesión se mantiene de la misma anchura si } \\
\text { transiluminamos / sombra gris fina en el esmalte }\end{array}$ \\
\hline $\begin{array}{l}\text { Amplia sombra gris en el esmalte cuando } \\
\text { transiluminamos }\end{array}$ \\
\hline $\begin{array}{l}\text { Ancha sombra gris en el esmalte sin evidencia de } \\
\text { sombra de dentina }\end{array}$ \\
\hline $\begin{array}{l}\text { Sombra naranja / marrón / azulada / negra menor a } 2 \\
\text { mm de ancho (dentina) }\end{array}$ \\
\hline $\begin{array}{l}\text { Sombra naranja / marrón / azulada / negra mayor a } 2 \\
\text { mm de ancho (dentina) }\end{array}$ \\
\hline $\begin{array}{l}\text { Amplia zona de cavitación franca con probable } \\
\text { participación de la pulpa }\end{array}$ \\
\hline
\end{tabular}

Tabla 3. Códigos radiográficos ICDAS:

\begin{tabular}{|c|l|}
\hline E0 & No hay lesión radiolúcida \\
\hline E1 & $\begin{array}{l}\text { Una lesión de caries en una } \\
\text { superficie proximal con forma de V ó U en la } \\
\text { Radiografía, dentro del esmalte y no alcanza } \\
\text { a la dentina. }\end{array}$ \\
\hline E2 & $\begin{array}{l}\text { Una lesión de caries en proximal aparece en } \\
\text { forma de U ó V en la Radiografía, ha } \\
\text { alcanzado o pasado ligeramente el límite } \\
\text { esmalte-dentina. }\end{array}$ \\
\hline D3 & $\begin{array}{l}\text { Una lesión de caries en una superficie } \\
\text { proximal que radiográficamente llega afectar } \\
\text { la dentina. }\end{array}$ \\
\hline D4 & $\begin{array}{l}\text { Una lesión de caries en una } \\
\text { superficie proximal, que radiográficamente } \\
\text { ha alcanzado la pulpa. }\end{array}$ \\
\hline
\end{tabular}

\section{RESULTADOS}

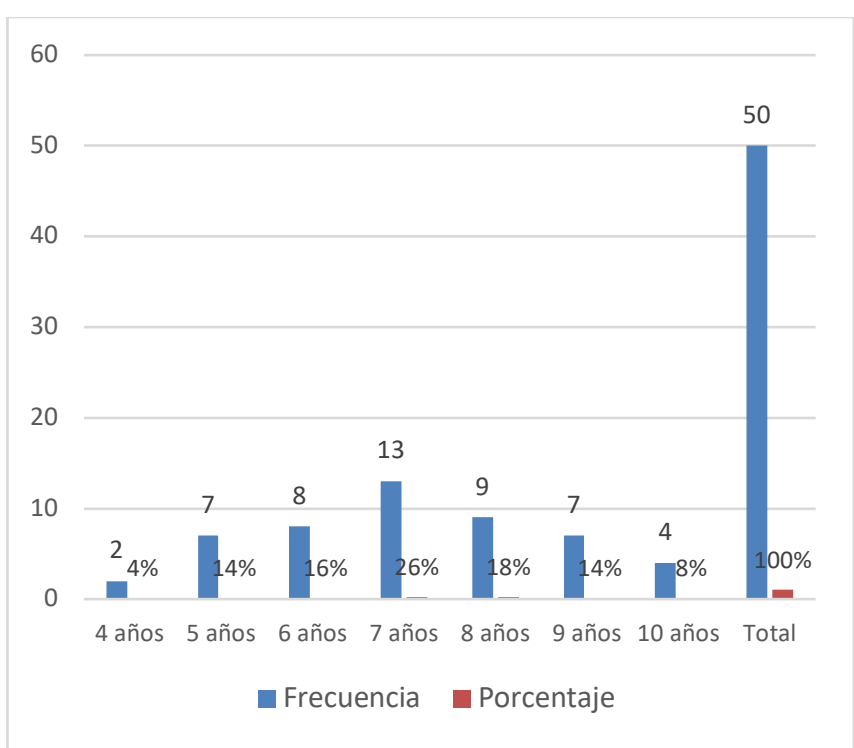

Figura 1 Edad de los niños-as de la Escuela de Educación Básica Mons. "Juan María Riofrío"

De los 50 niños-as seleccionadas para el diagnóstico respectivo, el $4 \%$ corresponde a 2 niños de 4 años, el $14 \%$ a 7 niños de 5 años, el $16 \%$ a 8 niños de 6 años, el $26 \%$ a 13 niños de 7 años, el $18 \%$ a 9 niños de 8 años, el $14 \%$ a 7 niños de 9 años y el $8 \%$ restante a 4 niños de 10 años. Todos los niños-niñas de la muestra presentaron molares deciduos en boca.

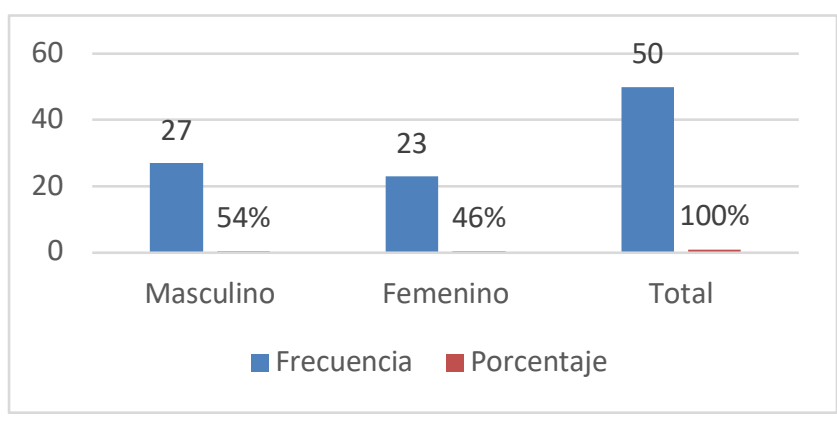

Figura 2 Sexo de los niños de la Escuela de Educación Básica Mons. "Juan María Riofrío"

En cuanto al sexo el $54 \%$ corresponde al sexo masculino con una frecuencia de 27 niños, mientras que el $46 \%$ restante corresponde al sexo femenino con una frecuencia de 23 niñas. 
Tabla 4. Código ICDAS según la Técnica de Diagnóstico Visual simple en las superficies proximales de molares deciduos

\begin{tabular}{|l|r|r|r|}
\hline & Sin caries & \multicolumn{3}{|c|}{ Caries incipiente } \\
\hline $\mathbf{N}^{\circ}$ Pieza & Código 0 & Código 1 & Código 2 \\
\hline $\mathbf{5 . 5}$ & 35 & 2 & 0 \\
\hline $\mathbf{5 . 4}$ & 35 & 2 & 0 \\
\hline $\mathbf{6 . 4}$ & 33 & 1 & 0 \\
\hline $\mathbf{6 . 5}$ & 40 & 4 & 0 \\
\hline $\mathbf{7 . 5}$ & 34 & 0 & 8 \\
\hline $\mathbf{7 . 4}$ & 21 & 2 & 1 \\
\hline $\mathbf{8 . 4}$ & 32 & 0 & 2 \\
\hline $\mathbf{8 . 5}$ & 37 & 0 & 2 \\
\hline Total & 267 & 11 & 13 \\
\hline
\end{tabular}

Mediante la aplicación de la Técnica diagnóstico visual simple, según el criterio ICDAS, se obtuvieron los siguientes resultados: 267 superficies proximales con código 0 que significa sanas al secado con aire durante 5 segundos; 11 superficies proximales con código 1 con mancha blanca marrón en esmalte seco y 13 superficies proximales con código 2 con mancha blanca marrón en esmalte húmedo.

Tabla 5. Código ICDAS según la Técnica de Magnificación en las superficies proximales de molares deciduos

\begin{tabular}{|l|c|c|c|}
\hline & Sin caries & \multicolumn{2}{|c|}{ Caries incipiente } \\
\hline $\mathbf{N}^{\circ}$ Pieza & Código 0 & Código 1 & Código 2 \\
\hline $\mathbf{5 . 5}$ & 29 & 8 & 0 \\
\hline $\mathbf{5 . 4}$ & 31 & 6 & 0 \\
\hline $\mathbf{6 . 4}$ & 32 & 3 & 0 \\
\hline $\mathbf{6 . 5}$ & 32 & 12 & 0 \\
\hline $\mathbf{7 . 5}$ & 20 & 5 & 17 \\
\hline $\mathbf{7 . 4}$ & 18 & 4 & 2 \\
\hline $\mathbf{8 . 4}$ & 30 & 0 & 4 \\
\hline $\mathbf{8 . 5}$ & 31 & 2 & 6 \\
\hline Total & 223 & 40 & 29 \\
\hline
\end{tabular}

Mediante la aplicación de la Técnica de magnificación diagnosticadas en las superficies proximales, según el criterio ICDAS, se obtuvieron los siguientes resultados: 223 superficies proximales con código 0 lo que significa sanas al secado con aire durante 5 segundos; 40 superficies proximales con código 1 con mancha blanca marrón en esmalte seco y 29 superficies proximales con código 2 con mancha blanca marrón en esmalte húmedo.

Tabla 6. Código ICDAS según la Técnica de Transiluminación en las superficies proximales de molares deciduos

\begin{tabular}{|l|r|r|r|}
\hline & \multicolumn{1}{|c|}{ Sin caries } & \multicolumn{3}{|c|}{ Caries incipiente } \\
\hline$N^{\circ}$ Pieza & Código 0 & Código 1 & Código 2 \\
\hline 5.5 & 35 & 2 & 0 \\
\hline 5.4 & 36 & 1 & 0 \\
\hline 6.4 & 34 & 0 & 0 \\
\hline 6.5 & 44 & 0 & 0 \\
\hline 7.5 & 37 & 5 & 0 \\
\hline 7.4 & 24 & 0 & 1 \\
\hline 8.4 & 34 & 0 & 0 \\
\hline 8.5 & 39 & 0 & 0 \\
\hline Total & 283 & 8 & 1 \\
\hline
\end{tabular}

Al evaluar las lesiones cariosas de las superficies proximales, diagnosticadas por la técnica de transiluminación, según el criterio ICDAS, se obtuvieron los siguientes resultados:

- 283 superficies proximales con código 0 lo que significa que no hay sombra o área manchada.

- 8 superficies proximales con código 1 que significa lesión se mantiene de la misma anchura si transiluminamos/sombra gris fina en el esmalte.

- 1 superficie proximal con código 2 lo que significa amplia sombra gris en el esmalte cuando transiluminamos. 
Tabla 7. Código ICDAS según la Técnica Radiográfica en las superficies proximales de molares deciduos

\begin{tabular}{|l|r|r|}
\hline & Sin caries & \multicolumn{2}{l|}{ Caries incipiente } \\
\hline $\mathbf{N}^{\circ}$ Pieza & E0 & E1 \\
\hline 5.5 & 39 & 0 \\
\hline 5.4 & 37 & 0 \\
\hline 6.4 & 33 & 2 \\
\hline 6.5 & 43 & 1 \\
\hline 7.5 & 39 & 3 \\
\hline 7.4 & 22 & 4 \\
\hline 8.4 & 35 & 0 \\
\hline 8.5 & 37 & 3 \\
\hline Total & 285 & 13 \\
\hline
\end{tabular}

Al evaluar las superficies proximales de los molares deciduos, mediante la Técnica radiográfica, según el criterio ICDAS, se obtuvieron los siguientes resultados: 285 superficies proximales con código E0 lo que significa que no hay sombra radiolúcida y 13 superficies proximales con código E1 que significa lesión de caries en una superficie proximal con forma de $\mathrm{V}$ ó $\mathrm{U}$ en la radiografía, dentro del esmalte y no alcanza a la dentina.

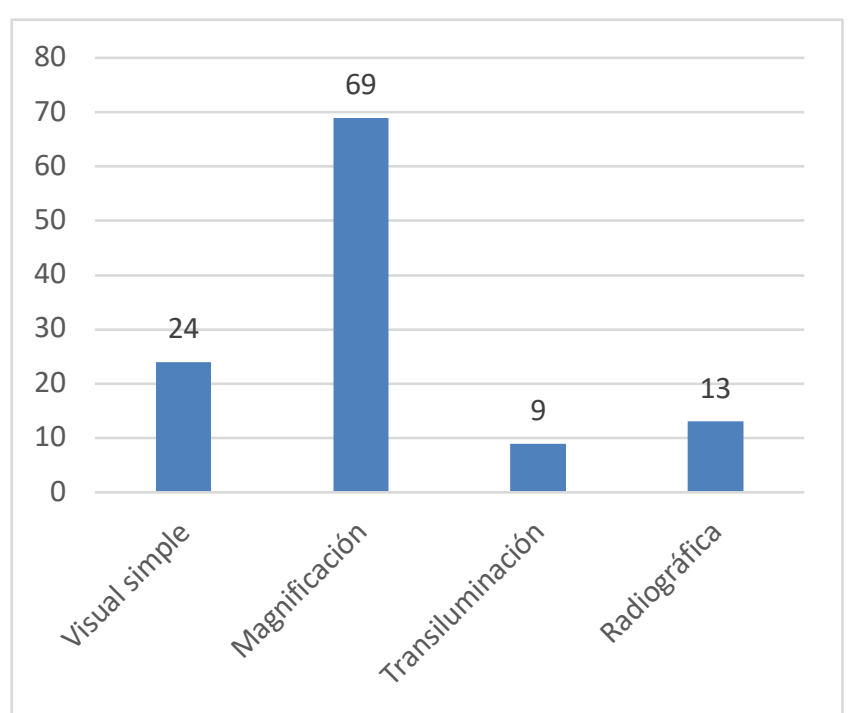

Figura 3 Superficies con caries incipiente interproximal en molares deciduas diagnosticadas por las cuatro técnicas
Al evaluarse la relación entre las cuatro técnicas de diagnóstico visual simple, magnificación, transiluminación y radiográfica de caries incipiente interproximal en molares deciduos, se puede determinar que la técnica de magnificación permite detectar mayor número de lesiones cariosas incipientes interproximal, siendo un total de 69 superficies interproximales. Seguidamente la técnica visual simple se detectó 24 superficies con caries incipientes interproximales; con la técnica radiográfica se diagnosticó 13 superficies con caries incipientes interproximales y finalmente se puede observar que mediante la técnica de transiluminación se detectó un menor número siendo 9 superficies con lesiones cariosas incipientes interproximales, según el criterio ICDAS.

Tabla 8. Frecuencia de las lesiones cariosas incipientes interproximales diagnosticadas por la técnica visual simple según su grado de actividad

\begin{tabular}{|c|c|c|}
\hline $\mathbf{N}^{\circ}$ Pieza & Activa & Inactiva \\
\hline $\mathbf{5 . 5}$ & 0 & 2 \\
\hline $\mathbf{5 . 4}$ & 0 & 2 \\
\hline $\mathbf{6 . 4}$ & 0 & 1 \\
\hline $\mathbf{6 . 5}$ & 1 & 3 \\
\hline $\mathbf{7 . 5}$ & 6 & 2 \\
\hline $\mathbf{7 . 4}$ & 0 & 3 \\
\hline $\mathbf{8 . 4}$ & 1 & 1 \\
\hline $\mathbf{8 . 5}$ & 1 & 1 \\
\hline Total & 9 & 15 \\
\hline
\end{tabular}

Del total de lesiones incipientes interproximales en molares deciduos diagnosticadas por la técnica visual simple siendo 24, según su grado de actividad se obtuvo como resultados: 9 activas con superficie rugosa-opaca y 15 superficies inactivas lisas-brillantes (Tabla 8).

Del total de lesiones incipientes interproximales en molares deciduos diagnosticadas por la técnica de magnificación siendo 69, según su grado de actividad se obtuvo como resultados: 29 activas con superficie rugosa-opaca y 40 superficies inactivas lisas-brillantes (Tabla 8 ) . 
Tabla 9. Frecuencia de las lesiones cariosas incipientes interproximales diagnosticadas por la técnica de magnificación según su grado de actividad

\begin{tabular}{|c|c|c|}
\hline $\mathbf{N}^{\circ}$ Pieza & Activa & Inactiva \\
\hline 5.5 & 1 & 7 \\
\hline 5.4 & 3 & 3 \\
\hline 6.4 & 1 & 2 \\
\hline 6.5 & 3 & 9 \\
\hline 7.5 & 14 & 8 \\
\hline 7.4 & 2 & 4 \\
\hline 8.4 & 2 & 2 \\
\hline 8.5 & 3 & 5 \\
\hline Total & 29 & 40 \\
\hline
\end{tabular}

La presente investigación se debe a la dificultad en el diagnóstico precoz de lesiones cariosas incipientes interproximal debido a su ubicación por lo que es difícil y / o impide examen clínico adecuado. Después de analizar los resultados del presente estudio, se puede afirmar que sí existe una relación significativa entre las técnicas de diagnóstico de caries incipiente interproximal en molares deciduos aplicadas a los niños de 4 a 10 años de edad.

Este estudio reveló que la técnica de magnificación $3,5 \mathrm{X}$ permite detectar mayor número de lesiones cariosas incipientes interproximales (mancha blanca marrón), seguidamente fue la técnica visual simple. Estos resultados coinciden con lo reportado por Gonzales y col (5)., en el año 1999 , quienes compararon la magnificación $3,25 X$ con otras técnicas de diagnóstico, concluyendo que este método presentaba la mayor eficacia. Lussi(6) comparó diferentes métodos de diagnóstico de caries, concluyendo que la inspección visual con magnificación $2 \mathrm{X}$ y la radiografía convencional presentaban la mayor sensibilidad para detectar caries incipientes. Estos datos coinciden con estudios realizados por Lavonius, Kerosuo, \& Kallio(7) quienes compararon los métodos de inspección visual, magnificación y transiluminación, concluyendo que la variación era mayor al comparar al primero con los dos últimos que comparando los dos últimos entre sí.

Mientras que Mitropoulos(10), comparó la transiluminación con fibra óptica y las radiografías bitewing para detectar caries interproximales, encontrando que la transiluminación ofrecía mejores resultados debido a que las radiografías bitewing producen sobreposición de imágenes a nivel de esmalte. Otros autores como Perez A.(11), en su estudio se diagnosticaron, mediante la técnica visual, 73 dientes (53\%) como sanos y $65(47 \%)$ con caries incipiente interproximal. Radiográficamente, 97 (70\%) fueron diagnosticados como sanos mientras que 41 (30\%) fueron diagnosticados con caries interproximal incipiente.

Los resultados nos permiten llegar a las siguientes conclusiones:

- La técnica visual simple es el método más utilizado y no requiere demasiado entrenamiento clínico. Sin embargo, presentó una sensibilidad media para el diagnóstico de lesiones cariosas incipientes.

- La magnificación con una lupa tipo visera de 3,5X permite realizar un diagnóstico de alto nivel de precisión en las diferentes especialidades.

- La técnica radiográfica aleta de mordida y de transiluminación con LED no son fiables para detectar caries incipiente interproximal, siendo más utilizadas y que complementan el diagnóstico de caries en una superficie proximal que llega afectar a dentina o a pulpa.

- Finalmente, al evaluar el grado de actividad de las lesiones cariosas interproximales, la técnica de magnificación $3.5 X$ resultó la más eficaz la cual permitió diagnosticar las superficies activas con superficie rugosaopaca y las superficies inactivas lisas-brillantes.

Se recomienda realizar investigaciones con una muestra mayor para aumentar así la credibilidad de los resultados, con utilización del índice ICDAS.

Así mismo es importante para hacer un diagnóstico correcto de lesiones cariosas en su etapa inicial, realizar una profilaxis dental previa para eliminar todos los restos alimenticios acumulados en los espacios interproximales, visualizar con mucha atención lesiones cariosas en las superficies proximales y complementarse con diferentes técnicas como magnificación, radiográficas, transiluminación.

Contribuciones de autoría: JPS y TVB diseñaron el estudio, participaron en la recolección de datos análisis e interpretación de resultados. Redactaron el artículo y aprobaron la versión final del artículo.

Fuente de financiamiento: El estudio fue financiado por los autores.

Conflicto de intereses: Los autores declararon no tener conflicto de interés.

\section{REFERENCIAS}

1. Barbería E. Odontopediatría. Barcelona: Masson S.A; 2da edición. 2001; 173-175.

2. Francia - Pinto C, Cenci E, Azevedo E, Goettems M, Torriani D, \& Romano A. Caries proximales en dientes primarios posteriores: diagnóstico y los factores asociados. Odontopediatría Clínica Integral. Brasil; 2011; 387-392. 
3. Bordóni N., Escobar A., Castillo R.,Odontologia Pediátrica. La salud Bucal del Niño y adolescente en el mundo actual. Ed Medica Panamericana; 2010. 549-584

4. González, M., Balda, R., \& al. Estudio comparativo de tres métodos de diagnóstico de caries. Acta odontol. venez; 2000: 53-80.

5. Lussi, A. Comparison of different methods for the diagnosis of fissure caries without cavitation. Caries Res; 2004: 409.

6. Lavonius, E., Kerosuo, E., \& Kallio, P. Occlusal restorative decisions based on visual inspectioncalibration and comparision of different methods. Community Dent Oral Epidemiol; 2003: 156-159.

7. Garcés, S. Estudio comparativo de tres métodos auxiliares para el diagnóstico de lesiones cariosas incipientes. Kiru; 2009: 27-35

8. Peers, A., Hill, F., Mitropoulos, C., \& al,. Validity and reproducibility of clinical examination, fibre-optic transillumination, and bite-wing radiology for the diagnosis of small approximal carious lesions: an in vitro Study. Caries Res; 2000: 307-311

9. Mitropoulos, C. Comparison of fibre-optic transillumination with bitewing radiographs. Rev British Dental Journal; 2001: 21-23

10. Perez, A. Capacidad diagnóstica de la fluorescencia láser para el diagnóstico de caries oclusal en dientes deciduos. Rev Estomatol Herediana; 2004: 5-11.

11. Harris N. Odontología Preventiva Primaria. México: Manual Moderno; 2012: 33-52

12. Ketley $\mathrm{CE}, \mathrm{H}$. R. Visual and radiographic caries in first permanent molars and in second primary molars. Br Dent J,; 1999; 364-70

13. Perez, M., Limeres, J., \& Javier, F. Manual de Higiene Oral. Santiago de Compostela: Idea Grafica Profesional; 2012

14. Shivakumar, K., Sumanth, P., \& Chandú, G. International Caries Detection and Assessment System: A new paradigm in detection of dental caries . J Conserv Dent; 2009: 10-16.

15. Verdonschot, E., Bronkhorst, E., \& Wenzel, A. Approximal caries diagnosis using fiber- optic transillumination: a mathematical to improve validity. Community Dent Oral Epidemiology: 2001; 329-332.

16. Vergara $\mathrm{C}$, Uribe $\mathrm{S}$. Evaluación in vitro de la resistencia compresiva de un sellante resinoso fluorado pre y post liberación de flúor. Rev. Clin. Periodoncia Implantol. Rehabil. Oral [Internet]. 2012 Abr [citado 2019 Feb 19] ; $5(1): 9-12$. 